\title{
Conflictos discursivos: un estudio de Adiós, Ayacucho desde la perspectiva del análisis crítico del discurso
}

\author{
Milagros Mere Collazos \\ Universidad Nacional Mayor de San Marcos \\ milymere@gmail.com
}

\section{Resumen}

Analiza la novela Adiós, Ayacucho de Julio Ortega desde el análisis crítico del discurso (ACD) que permite observar los recursos utilizados por el narrador para evidenciar las diferencias sociales y demandas de los desfavorecidos representados en la novela, los que expresan un discurso claramente distinguible. Parte de la premisa: en las obras literarias, el discurso empleado por el narrador, brinda información sobre la historia relatada y visualiza la relación establecida entre los diferentes actores de una sociedad en un momento determinado. En efecto, los diversos modos de interacción entre los grupos sociales a través del tiempo han ocasionado que algunos adquieran más poder que otros, lo que se evidencia en y a través del uso del lenguaje.

Palabras clave: Análisis Crítico del Discurso, Lenguaje, Significado, Grupos sociales

\begin{abstract}
Analyzes the novel Goodbye, Ayacucho of Julio Ortega from the critical analysis of the Discourse (ACD) that allows to observe the resources used by the narrator to demonstrate the social differences and demands of the disadvantaged represented in the novel, those who express a clearly distinguishable discourse. Part of the premise: in the literary works, the discourse used by the narrator, provides information on the story told and visualizes the relationship established between the different actors of a society at a given time. Indeed, the various modes of interaction between social groups over time have caused some to acquire more power than others, which is evident in and through the use of language.
\end{abstract}

Keywords: Critical Analysis of Discourse, Language, Meaning, Social Groups 


\section{Conflictos discursivos: un estudio de Adiós, Agacueho desde la perspectiva del análisis erítico del discurso}

La novela Adiós, Ayacucho, publicada en 1986, narra la historia de Alfonso Cánepa, un campesino asesinado por la policía porque es acusado de ser un terrorista. Él, pese a haber muerto, narra la historia de su muerte y su posterior viaje a la ciudad de Lima. En el trayecto, conoce a varios personajes, con quienes discute sobre los diferentes problemas por los que atraviesa el Perú en esa época (década de los ochenta). El objetivo de su viaje es entregarle una carta al presidente de la República, Fernando Belaúnde. En ella, se incluyen los reclamos del muerto ante la situación de injusticia cometida contra los miembros de diferentes comunidades del país, representados en Alfonso.

Llama la atención el hecho de que el muerto, consciente de que lo está, asuma rasgos característicos de una persona viva. En este sentido, en la novela, se puede encontrar momentos en los que sostiene conversaciones con diferentes personas, quienes no se sorprenden al verlo. Cabe hacer aquí la observación de que la muerte se ha naturalizado hasta el punto de que el muerto pasa por vivo sin afectar la percepción de la gente. Revivir al personaje principal y que este asuma el rol de ser el narrador de la historia no es una simple coincidencia. La intención del narrador es realizar una denuncia social y, con la finalidad de realizar este reclamo, recurre al empleo de diversas estrategias discursivas.

\section{E1 Análisis Crítico del Discurso como propuesta teórica frente a los fenó- menos sociales}

El Análisis Crítico del Discurso (ACD) tiene como finalidad investigar los problemas sociales manifestados a través del discurso. Básicamente, se ha concentrado en estudiar situaciones de desigualdad y poder en la sociedad, además de explicar en qué sentido las estructuras del discurso trasluce los comportamientos sociales. 
El discurso se concibe como una práctica social a partir de la cual se puede reconocer de qué manera las instituciones sociales han marcado las características de su uso, pero, además, cómo este determina las relaciones sociales y la identidad de las personas de manera individual o grupal. Esta situación es descrita por Fairclough y Wodak (2000) de la siguiente manera:

El hecho de describir el discurso como práctica social sugiere una relación dialéctica entre un suceso discursivo particular y las situaciones, instituciones y estructuras sociales que lo enmarcan. Ahora bien, una relación dialéctica es siempre bidireccional: el suceso discursivo está moldeado por las situaciones, instituciones y estructuras sociales, pero a su vez les da forma. (p.367)

En otras palabras, el discurso se construye a partir de las circunstancias sociales, pero también puede determinarlas. Sin embargo, el discurso está relacionado, también, con la ideología, es decir, con "las representaciones mentales que forman la base de la cognición social, esto es, del conocimiento y actitudes compartidos de un grupo" (Van Dijk, 2000, p.56). La ideología se relaciona con las creencias, las cuales moldean las acciones y posiciones de un grupo con respecto a alguna cuestión social. Cabe mencionar que los productos o prácticas discursivas no solo ponen en evidencia la ideología compartida por un grupo, sino también podrían influir en reproducir los contenidos u opiniones manejadas en ese contexto, por ejemplo, las diferencias sociales, culturales y étnicas en una circunstancia social determinada.

Se debe destacar, también, que las circunstancias sociales involucradas en la producción del discurso constituyen lo que se denomina contexto, el cual es considerado un elemento clave dentro de la propuesta del ACD. El contexto está conformado por algunos elementos básicos que lo definen, como los participantes, el tiempo o el lugar donde se ha emitido el discurso. Por esta razón, no deben ser excluidos del análisis. En términos de Teun A. van Dijk (2000), el discurso se relaciona con el contexto de la siguiente manera: "El discurso se produce, comprende y analiza en relación con las características del contexto". (p. 32) Esto quiere decir que el discurso es un elemento imprescindible en cualquier investigación enmarcada en el ACD.

\section{Estructuras básicas de análisis}

Teun A. van Dijk (2003) presenta algunos elementos básicos para analizar el discurso: el plano del significado y el plano formal (texto-contexto).

\subsection{Plano del significado/texto}

En este primer nivel, se incluye la información extraída del texto, en otras palabras, el contenido encontrado a partir de los significados expuestos en el discurso. Para ello, se divide el análisis a nivel de significados globales y locales. En el nivel de 
los significados globales, se incluye la mención de los temas abordados en el texto y cómo son presentados. En el nivel de los significados locales, se analiza la forma en que se presenta la información, es decir, si se utilizan recursos lingüísticos que destacan u ocultan información. Este nivel se divide en significados locales de carácter implícito y explícito. En el primero, se analizan extractos del texto que ponen en evidencia datos que no se enuncian directamente, sino que son mostrados a partir de presuposiciones, ambigüedades u otros recursos. En cambio, en el nivel explícito, se reconoce cuáles son los significados referidos de manera directa y clara en el texto. Por ejemplo, se puede identificar qué tipo de palabras se utiliza para describir a una entidad determinada.

\subsection{Plano formal/texto-contexto}

Este segundo apartado está dividido en dos partes. Por un lado, destacan las estructuras formales sutiles, en las que se intenta mostrar el discurso ideológico inferido a partir de las construcciones plasmadas en el nivel de significado, es decir, se indaga por los parámetros empleados para construir el discurso. Por otro lado, en el nivel contextual, se pone en evidencia el origen de las ideas que posibilitaron la creación del acto discursivo. Esto se relaciona directamente con las situaciones sociales, históricas, políticas y económicas relacionadas con la producción del texto.

Estos elementos (plano del significado y plano formal) han sido analizados en investigaciones anteriores por Browne Sartori (2010) en el campo del análisis crítico del discurso aplicado al ámbito periodístico. Sin embargo, este tipo de análisis también puede aplicarse a otros tipos de texto, como el caso de las producciones literarias. Debido a ello, en el presente análisis de la novela Adiós, Ayacucho, se propone la aplicación de estos diferentes niveles. Al ser la novela un producto más extenso que una noticia, se incluye una mayor cantidad de datos $\mathrm{y}$, por lo tanto, su presentación es más amplia. Su objetivo principal es sistematizar los recursos discursivos empleados. A continuación, se presenta la propuesta de análisis.

\subsection{Primer nivel de análisis: plano del significado}

\subsubsection{Nivel temático: significados globales}

En esta primera parte, se abordan los temas generales del texto. Adiós, Ayacucho se presenta en forma de narración, enunciada por el personaje principal Alfonso Cánepa, víctima del conflicto interno, quien se traza el objetivo de viajar a Lima para recuperar parte de su cuerpo, que le fue arrebatado cuando un policía lo asesinó, porque, aparentemente, lo confundieron con un terrorista. Este personaje, muerto, pero con todas las capacidades de una persona viva, en su trayecto hacia esta ciudad 
conoce a diferentes personajes, de los cuales destacan el antropólogo y el Petiso. A través de las conversaciones con estas y otras personas, se describe la situación sociopolítica del Perú en la época de gobierno del presidente Belaúnde, quien se encuentra con Alfonso al final en un mitin, pero lo ignora y bota la carta que este le entrega, en la cual se evidencian los reclamos del muerto ante la situación de responsabilidad asignada a las autoridades. Los problemas nacionales mencionados en la novela son el accionar injusto de los policías, la ineficacia de las autoridades del Gobierno, la descripción negativa de los antropólogos, la ingenuidad del peruano y la presentación de una Lima hostil para los migrantes.

\subsubsection{Nivel de significados locales}

En este segundo nivel de análisis, nos concentramos en encontrar fragmentos en los que se destaque información relevante sobre algún eje temático en particular. Cabe mencionar que esta información puede presentarse de manera implícita o explícita. En el análisis, luego de encontrar construcciones textuales de ambos tipos, las presentamos organizadas en ejes temáticos.

\section{a) Primer eje temático: el accionar injusto de los policías}

\section{-Significados de carácter implícito}

Yo sabía que me acusarían de terrorista, pero ellos sabían que yo no lo era, entonces, ¿qué querían que confiese? (p.67)

A partir del enunciado emitido por el narrador, se presupone que la policía realiza acusaciones falsas, lo que podría ocasionar un castigo injusto.

Solo un tonto podía creer tanto en los recursos legales. Solo yo, sabiendo que me acosaban, tenía que cruzar esa plaza y entrar a la comisaría. (p.69)

A partir de este segundo fragmento y relacionándolo con el anterior, se puede deducir que la policía no respeta la legalidad ni la formalidad. El narrador da a entender que esa situación es conocida por todos o, por lo menos, por la mayoría de los peruanos.

\section{-Significados de carácter explícito}

Me dije que tendría que llevar la cuenta precisa de mis partes perdidas para recobrarlas luego y darme sepultura; pero cuando me arrojaron por fin a un hueco ancho y poco profundo y empezaban a taparme con piedras y paja, he creído ver a uno de los policías hurgando en torno con una bolsa de plástico en las manos, y de inmediato he sabido que este hijo de mala madre recogería mis pedazos para llevarse medio cuerpo mío. (p.68) 
En este fragmento, se presentan acciones negativas de la policía a través de la inclusión de frases verbales, como las siguientes: "me arrojaron por fin a un hueco ancho", "este hijo de mala madre recogería mis pedazos para llevarse medio cuerpo mío". Por otro lado, destaca el uso de una frase ofensiva para el miembro de la policía al que se hace referencia en la narración: "hijo de mala madre".

Este mismo policía, antes de llegar al hueco que sería mi tumba, me ha rellenado la barriga con paja seca, riéndose de mí, como si yo fuese un muñeco hecho para ser deshecho. (p.68)

En este caso, también se emplean frases verbales para mostrar los hechos negativos realizados por un miembro de la policía. Esto se puede ver en el siguiente extracto: "me ha rellenado la barriga con paja seca, riéndose de mí." Esta acción es una evidencia de la intención que tienen los policías de ocultar las pruebas del crimen.

¿Qué parte de mí será la que me falta? Ese hijo de su madre, misti borrachoso, que llevaba mis huesos en una bolsa de plástico, ¿a dónde la llevaría? A dónde si no a Lima, panteón de pobres. (pp. 69-70)

Se presenta una imagen negativa del policía a través del uso de frases y adjetivos ofensivos, como "misti borrachoso" o "hijo de su madre". La palabra misti hace referencia al "blanco-mestizo", a partir de lo cual se infiere que Alfonso es de procedencia indígena.

\section{b) Segundo eje temático: la ineficacia de las autoridades del Gobierno}

\section{-Significados de carácter implícito}

De Lima están viniendo, dice la radio — dijo una voz que no reconocí-. Protestas hay por todas partes, dicen.

-Ya no hay remedio — dijo el viejo lechero—. Todo se borra cuando mata el gobierno. (p.71)

El sustento de la afirmación "Todo se borra cuando mata el gobierno" es la presuposición de que el Gobierno realiza acciones reprochables, pero que no son juzgadas ni sancionadas. El verbo borrar implica desaparecer o eliminar. Con ello, se infiere que el Gobierno tiene la potestad de borrar (delitos, pruebas, acciones, palabras); en cambio, los campesinos, no. Sin embargo, en la novela, la prueba del delito no logra ser borrada por las autoridades, ya que Alfonso mismo es la evidencia del asesinato cometido.

-Te irás en el próximo avión llevándote este maletín —me explicó el nuevo patriota - En el aeropuerto de Lima serás escoltado por las fuerzas antidrogas hasta el hangar oficial donde te esperará el narcodiputado Pastana con 
un maletín. Tendrás que llevar ese maletín con mucho cuidado a la aduana internacional, donde te esperará el ministro Proxy, quien sale esta noche a Ginebra para abrir, con ese dinero, una agencia de adopción de niños peruanos. ¿Entendido? (p.101)

Se presupone que las autoridades descritas por Alfonso Cánepa en este extracto mantienen vínculo con el narcotráfico. Este hecho es sustentado debido al uso del término "narcodiputado", palabra compuesta formada a partir de la unión de las palabras "narco" y "diputado". Por otro lado, se presenta la frase "abrir, con ese dinero, una agencia de adopción de niños peruanos", lo que mostraría, con completa naturalidad, la existencia de situaciones de corrupción. Asimismo, mediante la frase "serás escoltado por las fuerzas antidrogas" se evidencia que las fuerzas armadas también están al servicio del narcotráfico.

- Belaúnde echará un discursito sobre la necesidad de la caridad cristiana — dijo el Petiso- Somos un público conmovedor, ¿la manyas?

—iQué suerte! —exclamé-. Podré darle mi carta personalmente. Los tres ahogaron otra risotada. (p.119)

Del extracto anterior, se puede inferir que las autoridades emplean mensajes "artificiales" acerca de la población menos favorecida. Se brinda un apoyo aparente a este grupo de personas, porque ello genera beneficios. En otras palabras, se utiliza al pueblo para elaborar un discurso político. Así, se cumple la conocida frase "Todo por el pueblo, pero sin el pueblo" que alude a que "aparentemente" el Gobierno debe velar por el bienestar del pueblo, pero considera que este no tiene injerencia alguna en la toma de decisiones. Por esta razón, cuando Alfonso menciona que le dará la carta a Belaúnde, los demás se burlan de él, pues la gente ya no cree en la proximidad o confianza que pueda haber entre las autoridades y el pueblo.

Por fin, Belaúnde en persona y con el brazo en alto avanzó al centro de su escolta y se detuvo exactamente frente a mí. Lo vi agobiado, ceniciento, mortal. Lo miré sin emoción. Allí estaba el culpable de mi muerte, pero seguramente ignoraba hasta mi nombre, y tendría una explicación para probar su inocencia personal. Era, claro, un político. (p.119)

El análisis detenido del fragmento anterior remite a la presuposición de que las autoridades son declaradas inocentes de los delitos por los que son acusadas debido a su condición política. Esto quiere decir que el político, en el Perú, evade su responsabilidad frente a los actos negativos cometidos por sus subordinados. Es así que sus decisiones se transforman en actos de violencia, por los cuales se convierte en el responsable intelectual, no material. Por lo mencionado anteriormente, se deduce que el político, en la sociedad peruana, está libre de culpas. 


\section{-Significados de carácter explícito}

Me recomponía ayudado por el Petiso, cojeando y tosiendo, cuando en el piso oscuro he visto mi carta, arrugada y sin abrir. Belaúnde, simplemente, había decidido no leerla. (p.120)

En este caso, la frase "Belaúnde, simplemente, había decidido no leerla", presenta, de manera directa, a través de una frase verbal, la descripción de la negativa del presidente ante una solicitud de un ciudadano indígena, serrano, víctima y con necesidad de ser atendido.

\section{c) Tercer eje temático: la descripción negativa de los antropólogos}

\section{-Significados de carácter implícito}

Estos antropólogos limeños son muy temperamentales. Un día se visten de indios y mastican coca, y al otro día vienen con la policía a enterrarnos a todos. (p.76)

El fragmento anterior muestra la idea de que los antropólogos actúan según su conveniencia: cuando necesitan de la población campesina, se unen a sus costumbres y creencias; sin embargo, cuando no la necesitan, simplemente la traicionan. Parte de la metodología de trabajo de campo de la antropología consiste en mimetizarse con el otro (persona objeto de estudio) a través de actividades como la comida o la vestimenta con la finalidad de obtener lo deseado: recopilar información para la investigación realizada.

- Sí, hombre, ¿no ves que a nombre de la autoridad se promete la justicia cuando se está reafirmando el poder estatal? ¿No crees que con ese discurso de Uchuracay termina la antropología en el Perú? (p.77)

El discurso de Uchuracay ${ }^{1}$ es el documento que ocasiona que la población campesina ya no confíe más en la labor de los antropólogos, pues el mensaje incluido no es objetivo; es decir, no se brinda información precisa acerca de los hechos ocurridos. A partir del extracto anterior, se infiere que la antropología como disciplina ha fracasado para explicar la realidad peruana, ya que distorsiona la realidad y está al servicio del discurso oficial.

Me puse a cantar un huayno de mi tierra. Álex me acompañaba, alegre de ver al antropólogo, por primera vez, acorralado. También él había entendido que el otro era un pishtaco disfrazado de doctor. (p.91)

1 El Informe de Uchuraccay se elaboró para informar acerca de los hechos relacionados con la muerte de ocho periodistas en la comunidad de Uchuraccay, Huanta, Ayacucho. Sin embargo, se afirma que los contenidos incluidos en ese documento no fueron totalmente objetivos, ya que se califica indirectamente a la población de dicha comunidad a partir de criterios como la falta de modernidad. 
El narrador intenta poner en duda la capacidad profesional del antropólogo, lo que se evidencia con la frase "disfrazado de doctor". Asimismo, emplea la analogía pishtaco $=$ antropólogo. Considerando que el pishtaco es un personaje mitológico que degüella a sus víctimas para extraerles la grasa y venderla, se destaca la característica de búsqueda de beneficio a costa de una víctima. Este es el concepto que se tiene de la labor de un antropólogo.

\section{-De carácter explícito}

De estas cosas venía charlando con el tipo raro que me asustó en el camión, y que resultó ser un estudiante de antropología, limeño, blanquito y criollo. (p.73)

El uso de adjetivos como "limeño", "blanquito" y "criollo" sirven para expresar las condiciones comunes de un antropólogo o estudiante de antropología de esa época. De ello se deduce que el sector que tenía mayores posibilidades de acceder a la educación universitaria, entonces, era aquel que presentaba mayor capacidad adquisitiva, caracterizado, principalmente por los rasgos físicos propios de los criollos pudientes.

\section{d) Cuarto eje temático: Lima: una ciudad hostil para los migrantes}

\section{-De carácter implícito}

Bajo el cielo de ceniza y sorteando charcos en esta tierra muerta, volví a sentir esa característica desolación que solo en Lima uno siente, y que oprime el corazón como un puño debilitado. (p.105)

Mediante la frase "tierra muerta" se describe una ciudad estéril, que no produce. Esta frase se sustenta a partir de la poca producción de alimentos en la costa a diferencia de la sierra. Asimismo, en el mismo fragmento, se menciona la desolación que se siente en la ciudad de Lima, lo cual se refiere a la situación de desamparo y de nostalgia de los migrantes al encontrarse en una ciudad que les brinda pocas posibilidades de desarrollo.

\section{-De carácter explícito}

¿Tendría que descubrirme llegando a Lima? La gente de Lima estaba acostumbrada a ver cadáveres en la televisión. En cuanto lograse yo hacerme entender, esa gente estaría de mi lado, y reclamarían para mí la parte de cadáver que la policía me ha quitado. No faltarían voluntarios para enterrarme a prisa. (pp. 71-72)

En el extracto anterior, se emplea una frase verbal "Lima estaba acostumbrada a ver cadáveres en la televisión”. A través de ella, se indica que, durante esos años, las noticias de muertes eran frecuentes en la sociedad limeña, lo que causó, en algunos 
grupos, indiferencia ante los hechos de violencia. Es decir, la muerte ya no sensibiliza al limeño, pues esta se ha "naturalizado".

Al salir a la calle el intenso olor me resultó familiar. Lima olía imparcialmente a orines. (p.115)

A través de la frase verbal "Lima olía imparcialmente a orines", se describe una ciudad sucia. Específicamente, se hace alusión a una mala costumbre limeña: orinar en la calle. Este breve fragmento resume, en parte, la situación de desorden en la que se encontraba el país en esa época: acumulación de basura, falta de limpieza, caos en las calles, robos, entre otros problemas.

\section{e) Quinto eje temático: la ingenuidad del peruano}

\section{-Significados de carácter implícito}

[...] y me entretuve pensando en mi corta vida de peruano crédulo. Porque como dice mi compadre Juani ya es bastante con que uno sea de por aquí, pero encima que lo agarren de cojudo es demasiado. Pero así es uno de nacimiento, y en el bar "La Caleta", lamentando mi mala suerte, poniéndose serio, mi compadre dirá a los otros: "Es que era demasiado peruano", y todos entenderían, asintiendo, que yo era cojudo de nacimiento. (p. 68-69)

En este fragmento, mediante la frase "ya es bastante con que uno sea de por aquí" se infiere que ser peruano es equivalente a tener una vida heroica o tener mala suerte. Asimismo, el peruano se representa como una persona fácil de engañar. Ello se evidencia con la frase "cojudo de nacimiento". Entonces, ser peruano no se define con el uso de términos más específicos, como pertenecer a un territorio concreto, sino con una característica asumida debido a un rol al cual se tiene que someter: ser subordinado, es decir, alguien dependiente de otro con más poder, al cual le tiene que creer y obedecer.

Cuando escuché que alguien silbaba junto a mí no pude reprimir un sobresalto. Me descubrí y le vi la cara al hombre que silbaba a mi lado, y que dejaba de silbar y empezaba a abrir los ojos más y más.

—Perdón — dije, y me tapé como pude.

Hasta en eso se ve que uno es un muerto peruano, que anda pidiendo excusas, porque un muerto argentino o cubano habría echado al intruso sin contemplaciones. (pp. 72-73)

A partir de este extracto, se deduce que los peruanos son temerosos, sumisos, ensimismados, retraídos, avergonzados y, en su mayoría, incapaces de enfrentarse a los demás. Por esa razón, para los peruanos, es difícil intentar revertir la situación de conflicto social no solucionada por las autoridades. 
Es cierto que los peruanos mejoramos de muertos, porque somos irrefutables, públicos y, a veces, magníficos. (p.80)

Se presupone que la muerte es una condición a través de la cual el peruano logra ser valorado. En muchas situaciones, esta se ha convertido en un puente para destacar o reconocer características que no eran consideradas cuando la persona aún vivía. Así, muchos de los problemas del pueblo recién se ponen en evidencia después de la muerte.

Yo estaba preparado para lo peor, como buen campesino del Perú, pero perder mi buen nombre y ser denunciado por los cien periódicos de Belaúnde como narcotraficante, lo cual sin duda estaba planeado hace tiempo, me resultaba intolerable, y yo tendría que imaginar, una vez más, algún recurso honroso en tanta humillación. (p.103)

Mediante la frase "tendría que imaginar, una vez más, algún recurso honroso en tanta humillación”, se infiere que el peruano, pese a las adversidades, suele defender su honra y, en la medida de lo posible, no permite la humillación. Asimismo, el fragmento sugiere que el campesino peruano está sometido a la manipulación por parte de los grupos de poder. Esta situación se presenta como un hecho totalmente natural, lo que se evidencia a través de la frase "una vez más".

\subsection{Segundo nivel de análisis: plano formal}

\subsubsection{Estructuras formales sutiles}

En el texto, se presentan muchos problemas sociales en el Perú, pero, principalmente, se destaca cómo estos afectan a las poblaciones campesinas. Las clases de poder (política, intelectual) y los policías no actúan conforme a ley ni tienen principios éticos. La población menos favorecida no tiene alternativa más que aceptar y quedarse callada, situación que quiere revertir Alfonso Cánepa a través de la elaboración de su carta dirigida al presidente Belaúnde, quien lo ignora. Así, se muestra que las autoridades no prestaban atención a lo que ocurría con el pueblo. Solo se acercaban a los sectores menos favorecidos cuando necesitaban extraer algún beneficio de ello, situación que se ve ilustrada, también, en la descripción de la labor de los antropólogos.

El objetivo de Alfonso Cánepa es entregarle su carta al presidente Belaúnde y, además, recuperar parte de su cuerpo. El primer aspecto mencionado, indirectamente, hace referencia a la necesidad que tiene la gente de comunicarse con las autoridades, quienes no siempre son accesibles a la población. Por otro lado, a partir de lo analizado a nivel de todo el texto, los restos del cuerpo estarían representando los derechos perdidos de la población a la cual representa este personaje. Por esta razón, se justifica la terquedad de Alfonso para buscar al Presidente y entregar su carta, puesto que, con ello, se estaría intentando alterar 
la actitud "pasiva" que habría asumido la mayor parte de los sectores menos favorecidos. Estos, tradicionalmente, han sido vistos como seres de tercera categoría, que tenían derechos constitucionales teóricamente, pero que no se respetaban en la práctica.

\subsubsection{Nivel contextual}

En la década de los ochenta, el problema más grave que tuvo que enfrentar el país fue el surgimiento de Sendero Luminoso, lo que desencadenó una serie de hechos negativos, como la muerte de mucha gente. Ante esa situación, el Gobierno, liderado por el presidente Fernando Belaúnde Terry, tuvo que enviar a las fuerzas armadas y a la policía a distintas zonas del país para controlar el problema. Debido a ello hubo una serie de enfrentamientos y muchas personas inocentes perdieron la vida. Sin embargo, la postura adoptada por Belaúnde no fue tan drástica, por lo que generaba impaciencia y desconfianza en la población. Incluso, algunos miembros de su equipo de trabajo no tuvieron un buen desempeño, lo que ocasionó que los problemas se agudicen.

\subsection{Resultados generales}

En general, el narrador ha construido un discurso en el cual se ha manifestado una denuncia social a partir de un hecho que resulta ser el eje de toda la novela: la búsqueda de reclamo de un muerto a través de la entrega de su carta al presidente Belaúnde. Al respecto, en un estudio previo realizado por Víctor Vich y Alexandra Hibbett (2009) se remarcó la idea de que esta novela es un texto escrito en respuesta a la Comisión Uchuraccay. Aunque estamos de acuerdo con lo afirmado por estos investigadores, consideramos que la denuncia social propuesta por el narrador va más allá del informe de Uchuraccay; es decir, es un reclamo general a la dinámica social establecida en la década de los ochenta, puesto que no solo se hace mención a temas relacionados con el informe, sino que también se introducen otras temáticas que lo complementan.

Para describir los hechos y presentar la historia sin descuidar el objetivo, ${ }^{2}$ el narrador emplea formas lingüísticas que son funcionales con su rol como participante de la situación comunicativa y con lo que se quiere conseguir.

2 Víctor Quiroz, en su tesis La representación del conficto o el conflicto de la representación, Fricciones post/coloniales en Adiós Ayacucho y Lituma en los Andes (2013) menciona que "Adiós Ayacucho" es un texto con carácter carnavalizador, cuyo objetivo es subvertir las relaciones entre lo hegemónico y lo subalterno. (p.252) La afirmación del autor se complementa con los resultados arrojados en el presente análisis. 


\subsubsection{Plano significado/texto}

a) Nivel de significados globales: $\mathrm{El}$ texto hace referencia a varios temas relacionados con los problemas nacionales. Por ser el más mencionado y resaltado en la novela, además de vincularse directamente con el objetivo de la construcción del discurso por parte del narrador, el eje temático que destaca es el accionar incorrecto de las autoridades y la clase intelectual.

b) Nivel de significados locales: El narrador representa la sociedad peruana de la época a partir de la caracterización de diversos actores involucrados en el texto. El narrador, quien es el protagonista de la novela, configura los comportamientos sociales de la historia a la cual hacemos referencia. En cuanto a las temáticas desarrolladas a nivel de todo el texto, una de ellas está referida a las acciones de los policías. En ella, el nivel de significado más empleado es el de carácter explícito, mientras que, para describir el comportamiento del Gobierno y otras autoridades relacionadas con él, se emplea el nivel de significado implícito. Esto se puede interpretar desde dos direcciones. Por un lado, las comunidades están más acostumbradas a observar y juzgar las acciones que realizan los policías porque pueden comprobarlo en su vida cotidiana. No hay duda alguna de lo que el narrador afirma en el texto, de ahí el uso de significado explícito en la novela. En cambio, no se puede afirmar lo mismo de los miembros del Gobierno, ya que su accionar no es evidente y próximo a los pobladores campesinos. Se sabe que actúan de manera inapropiada, pero no es demostrado siempre. Por otro lado, el uso mayoritario de lo explícito para describir a los miembros de la policía, a diferencia de la descripción del Gobierno, se puede deber a que el narrador prefiere mantener, de alguna manera, el respeto por el orden jerárquico de poder. El máximo nivel le pertenece a las autoridades políticas, mientras que la policía ocupa un lugar menor, por ello, la libertad de poder utilizar el nivel explícito para referirse a ella.

Asimismo, en cuanto a la descripción de los antropólogos y del peruano, se utiliza con mayor frecuencia el nivel implícito. Solo el tema de la descripción de la ciudad de Lima incluye mayor cantidad de enunciados relacionados con el uso del significado a nivel explícito.

Por lo encontrado en el análisis de los enunciados referidos a estos temas, se puede afirmar que el principal recurso elegido por el narrador en el discurso es el de nivel implícito, el cual estaría relacionado con el objetivo básico: plasmar una denuncia ante las desigualdades sociales. No obstante, se emplea lo implícito o indirecto, debido a que, generalmente, el narrador, pese a realizar un reclamo, prefiere recurrir al respeto por las estructuras sociales. Por ello, se habla acerca de los problemas en el país, pero de manera indirecta u oculta. 


\subsubsection{Plano formal/texto-contexto}

a) Estructuras formales sutiles: Se presentan estructuras mentales negativas de los siguientes elementos de la sociedad: la policía, el Gobierno y los antropólogos. Primero, la policía es una institución que comete abusos de poder aduciendo que debe salvaguardar el orden en una situación de tensión social. Segundo, el Presidente actúa de modo indiferente ante la crisis social y política de aquel entonces. Por ello, la población afectada no siente que se cumplan sus derechos; tampoco se siente protegida y escuchada. Por último, en el texto, se evidencia una imagen mental negativa con respecto a los antropólogos, ya que son presentados como personas que solo buscan cumplir sus objetivos, incluso a costa de la población campesina. Como consecuencia de todo lo descrito, hay necesidad imperiosa de reclamo.

b) Nivel contextual: La raíz de toda la problemática acontecida en el país es el surgimiento de grupos subversivos como Sendero Luminoso. Las actitudes asumidas por las autoridades generaron disconformidad en la población. La relación entre estos hechos y la construcción del texto es referida en el estudio de Vich y Hibbett (2009): “[...] la novela muestra cómo el subalterno ${ }^{3}$ comienza a construir, por sí mismo, una explicación sobre la forma en que su identidad se encuentra inscrita en la realidad del país". (p. 182)

\section{Conclusiones}

El discurso del narrador en la novela Adiós, Ayacucho es construido con la finalidad de realizar una denuncia relacionada con las desigualdades sociales y el abuso de poder en la época de conflicto interno en la década de los ochenta. Para lograr su objetivo, el narrador, generalmente, recurre al uso de significados implícitos con la finalidad de evidenciar diversos problemas sociales como la ineficacia de las autoridades del Gobierno y el accionar incorrecto del sector intelectual (representado por el antropólogo). Cabe mencionar que el contenido referido a estos temas se manifiesta, principalmente, de manera indirecta, con lenguaje implícito, puesto que el narrador no quiebra totalmente el ordenamiento social, es decir, la jerarquía de poder establecida socialmente. Esto se conserva hasta el final de la novela, cuando se describe cómo Alfonso se conforma con ingresar a la tumba de Francisco Pizarro con la finalidad de completar los huesos de su cuerpo desintegrando los del conquistador. Asimismo, en la novela, se incluyen los siguientes temas: el accionar injusto de los policías, la ingenuidad del peruano y la presentación de Lima como una ciudad hostil para el migrante. El narrador, en todo el texto, emplea el discurso para realizar una crítica al oficialismo que actúa en desmedro de la población andina.

3 El individuo subalterno es aquel que pertenece a los sectores marginalizados en la sociedad. 
Se ha probado la efectividad del ACD para analizar e interpretar textos literarios. En efecto, en los mundos representados en la narrativa, se transcriben los discursos de los diferentes actores sociales que conforman la sociedad y se evidencian las relaciones de poder y subordinación que se manifiestan en y a través del discurso.

\section{Referencias bibliográficas}

Browne, R. \& Romero, P. (2010). Análisis Crítico del Discurso (ACD) de la representación boliviana en las noticias de la prensa diaria de cobertura nacional: El caso de El Mercurio y La Tercera. Polis (Santiago), 9 (26), 233-249. Recuperado el 05 de diciembre de 2015, de http://www.scielo.cl/scielo.php?script=sci_arttext\&pid=S0718-65682010000200012\&lng=es\&tlng=es. $\quad 10.4067 / \mathrm{S} 0718-$ 65682010000200012.

Fairclough, N. \&Wodak, R. (2000). Análisis crítico del discurso. En: Van Dijk, Teun. El discurso como interacción social. Estudios sobre el discurso II: Una introducción multidisciplinaria. (pp. 367- 404). Barcelona: Editorial Gedisa.

Ortega,J. (2006). Adiós, Ayacucho. En: Faverón, Gustavo. Toda la sangre. Antología de cuentos peruanos sobre la violencia politica. (pp. 67-122). Lima: Grupo Editorial Matalamanga.

Quiroz, V. (2013). La representación del conflicto o el conflicto de la representación, Fricciones post/coloniales en Adiós Ayacucho y Lituma en los Andes. Tesis presentada para optar el grado de Magíster en Literatura con mención en Literatura Peruana y Latinoamericana, Universidad Nacional Mayor de San Marcos, Lima.

Van Dijk, T. (2000). El discurso como interacción en la sociedad. En: Van Dijk, Teun. El discurso como interacción social. Estudios sobre el discurso II: Una introducción multidisciplinaria. (pp. 19-66). Barcelona: Editorial Gedisa.

Vich, V. \& Hibbett, A (2009). La risa irónica de un cuerpo roto: Adiós Ayacucho de Julio Ortega. En: Ubilluz, Juan Carlos, Hibbett, Alexandra \& Vich, Víctor. Contra el sueño de los justos: la literatura peruana ante la violencia política. (pp. 175-189). Lima: IEP. 
